\title{
The flux compression generator load parameters selection
}

\author{
Mirosław Wołoszyn ${ }^{1}$, Daniel Kowalak ${ }^{1}$, Kazimierz Jakubiuk ${ }^{1, *}$, Mikołaj Nowak $^{1}$ \\ ${ }^{1}$ Gdańsk University of Technology, Faculty of Electrical and Control Engineering, Narutowicza Str. 11/12, 80-233 Gdańsk, Poland
}

\begin{abstract}
Computer simulation results of the flux compression generator (FCG) loaded with an inductor has been presented in this paper. Simulation research has been performed in order to select the parameters of FCG load coil properly. The influence of the load inductance and resistance on the current gain factor and the magnetic field energy accumulated in a load coil has been investigated.
\end{abstract}

\section{Introduction}

Formation of the high current surges (in the order of MA) with a very short rise time (in the order of $\mu$ s) in the inductive load (induction coil) is necessary for the implementation of many scientific and technical research [2]. This type of current surge has been usually formed in Flux Compression Generators (FCG) $[1,2,3]$. The idea of the FCG operation has been shown in Fig. 1.

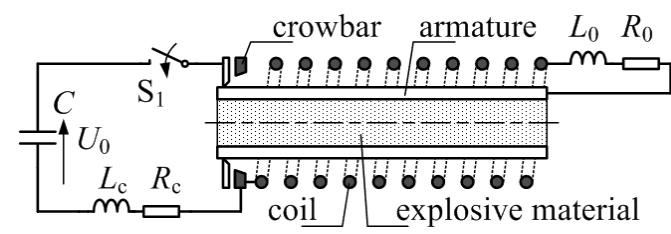

a)

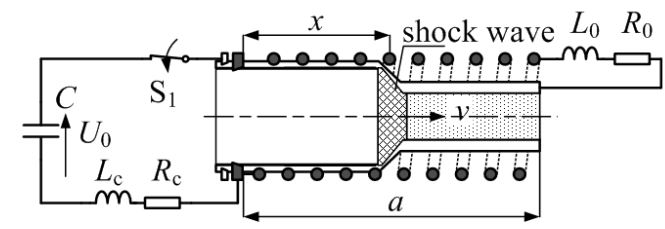

b)

Fig. 1. FCG operation idea: a) the initial state of the system, b) magnetic field compression caused by the armature expansion process, $C$ - capacitor bank charged to the voltage $U_{0}, L_{c}, R_{c}$ connections inductance and resistance, $L_{0}, R_{0}$ - load coil inductance and resistance.

The magnetic field is generated in FCG due to a current flow (in the order of $\mathrm{kA}$ ) through a properly shaped wiring system. At the moment when the value of the current from the primary source reaches the maximum value, the explosion of material located inside the wiring system is initiated, while the input terminals are shorted by crowbar. The explosion causes a reduction of an air volume between the wires and the compression of the magnetic field. As a result, the induction of the magnetic field increases rapidly and the current increases to the value of the order of MA [1, 3, 4, 5]. In spite of the fact that only a few percent of the energy stored in the explosives turns into the energy of the electromagnetic field, the enormous density of this energy reaches the desired effect [1].

\section{FCG general model}

The load coil parameters proper selection combined with the FCG model was under consideration. The view of the individual sections of the FCG coil has been presented in Fig. 2 [5].

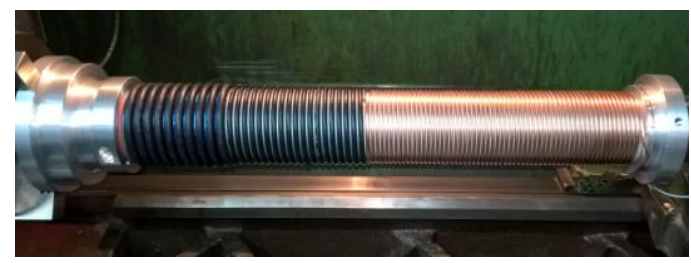

Fig. 2. View of the multi-section coil of the FCG model.

Fig. 3a shows the characteristics of the generator inductance $L_{g}$ which changes in three sections of the generator approximately linearly in a function of the dimensionless effective length of the winding.

The change of the FCG inductance $L_{g}$ occurs during the detonation process of the explosive located in the armature as a result of the shorting process of the individual turns of the coil.

The computer program has been developed to simulate the FCG current loaded with the load coil. The calculations have been performed for a different values of the load coil inductance $L_{0}$ and resistance $R_{0}$.

Due to the constant speed $v$ of shorting the individual turns of the generator coil, change of the FCG inductance $L_{g}$ as a function of the time can be determined by the formula [5]:

$$
\frac{d L_{g}}{d t}=\frac{d L_{g}}{d x} \frac{d x}{d t}=\frac{d L_{g}}{d x} v
$$

The characteristic of the FCG winding inductance $L_{g}$ as a function of the dimensionless, shorted part of the coil $x / a$ (Fig. 1b) calculated using the FEM method

* Corresponding author: kazimierz.jakubiuk@pg.edu.pl 
simulation has been presented in Fig. 3a. The FCG coil resistance $R_{g}$ is determined by a formula:

$$
R_{g}=\left(1-\frac{x}{a}\right) R_{g 0}
$$

where $R_{g 0}$ - the FCG coil initial resistance.

The FCG current generation calculations were performed in the circuit as shown in Fig. $3 b$.

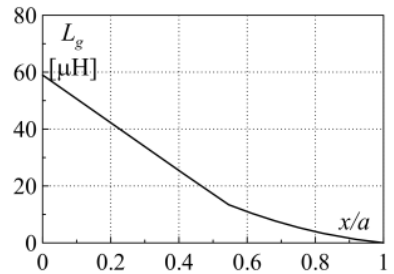

a)

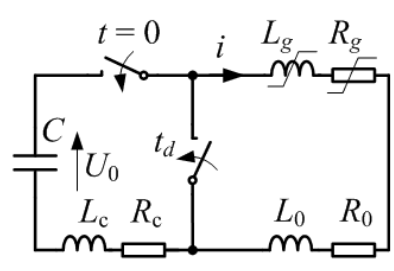

b)
Fig. 3. Characteristics of the FCG inductance $L_{g}$ as a function of its dimensionless length (a) and the parametric circuit of the FCG model (b).

The value of the current generated during field compression process has been calculated on the basis of the equation:

$$
i \frac{d L_{g}}{d x} v+\left(L_{g}+L_{0}\right) \frac{d i}{d t}+i\left(R_{g}+R_{0}\right)=0
$$

\section{Computational results}

The numerical simulations were performed on the basis of the computational model. The goal of the analysis was to determine the impact of FCG load parameters, i.e. inductance $L_{0}$ and resistance $R_{0}$, on the maximum values of generated current $i_{\max }$ and the amount of magnetic field energy $W$ accumulated in the load coil.

The dependence of the maximum value of the generated current $i_{\max }$ in the system as a function of the load inductance $L_{0}$ (for three values of the load resistance $R_{0}$ ) has been shown in Fig. 4a. Fig. 4b shows the dependence of the maximum magnetic field energy $W_{\max }$ accumulated in the load as a function of the load inductance $L_{0}$ for three values of the coil resistance $R_{0}$. Simulations have been performed for the assumed values of the other system parameters.

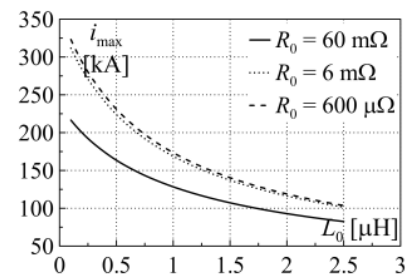

a)

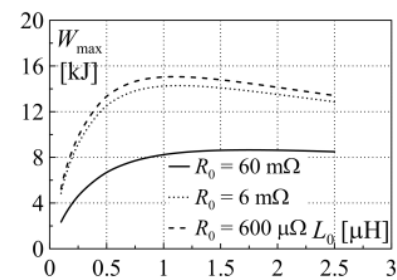

b)
Fig. 4. The characteristics of the: a) maximum value of the generated current $i_{\max }$ in the system as a function of the load inductance $L_{0}$ and b) the maximum energy $W_{\max }$ of the magnetic field accumulated in the load coil as a function of the inductance $L_{0}$ (for three values of its resistance $R_{0}$ ).

There is a value of the load inductance $L_{0}$ for which the energy accumulated in the magnetic field $W_{\max }$ reaches maximum value. Attention should be paid to the significant influence of the load coil resistance $R_{0}$ on the maximum value of the current $i_{\max }$ and the maximum energy of the magnetic field. It is therefore suggested to construct a load coil with a relatively large cross-section area wires. For a load coil resistance of a few $\mathrm{m} \Omega$, the optimal inductance exists and its value is about $1 \mu \mathrm{H}$.

The generator shorted on the output has a gain of about 44. The increase of the load inductance results in a significant reduction of the generated current $i_{\max }$.

\section{Conclusions}

Based on the presented simulation and experimental research following conclusions have been drawn:

- The current gain factor in the FCG system decreases non-linearly (approximately exponentially) as a function of the load inductance $L_{0}$.

- The additional phenomena decreasing the current gain coefficient in the system is the increase of resistance of the load coil $R_{0}$ as a result of its temperature increase and increase of a resistance as a result of the skin effect. These factors have not been studied in this paper.

- The maximum value of magnetic field energy $W_{\max }$ stored in the load coil exists for a specific load inductance value. Its value is also strongly influenced by the load resistance $R_{0}$. For larger resistance values, the $W_{\max }=f\left(L_{0}\right)$ characteristic becomes more flat which means that the effect of the system resistance is smaller.

The research has been performed as a part of the project financed by the Polish National Centre for Research and Development - contract DOB-1-1/1/PS/2014.

\section{References}

1 L. L. Altgilbers, et. al., Springer Verlag. New York, 2000

2 K. Jakubiuk, D. Kowalak, M. Nowak, ITM Web Conference, Volume 19, 2018 Computer Applications in Electrical Engineering (ZKwE'2018)

3 A. Neuber, Springer-Verlag. Berlin Heidelberg, 2005

4 M. Wołoszyn, K. Jakubiuk, P. Zimny, D. Kowalak, Archives of Electrical Engineering, Vol. 66, 2017

5 M. Wołoszyn, D. Kowalak, K. Jakubiuk, M. Nowak, Poznan University of Technology Academic Journal, Vol. 97(2019) 